\title{
A Virtual Input-Output Framework for Transceiver Analysis and Design for Multipath Fading Channels
}

\author{
Ashwin Ganesan and Akbar M. Sayeed, Senior Member, IEEE
}

\begin{abstract}
An understanding of the interaction between the channel and the signal space is key to reliable communication. Multipath fading channels exhibit inherent diversity that can be exploited via appropriate signaling and reception. In this paper, we develop a virtual multiple-input multiple-output framework for characterizing single-transmitter single-receiver multipath fading channels, where the virtual multiple inputs and outputs are created by dimensions of the signaling scheme. The essence of the framework is a representation of the system with respect to appropriately chosen basis waveforms for the signal space that expose the inherent structure of the channel. The structure makes it possible to derive closed-form expressions for ergodic and outage capacity for a variety of transceivers and to design novel transceivers. In many cases, the analysis clearly reveals the key factors that affect system performance. We provide new capacity expressions for a variety of transceivers. In particular, by analyzing particular transceivers, we provide new expressions that bound the outage capacity performance of the multipath channel. A novelty of this work lies in being able to apply codes developed for orthogonal frequency-division multiplexing systems and multiple antenna systems to existing code-division multiple-access-based systems.
\end{abstract}

Index Terms-Code-division multiple access (CDMA), multipleinput multiple-output (MIMO) systems, multipath fading, orthogonal frequency-division multiplexing (OFDM), outage capacity.

\section{INTRODUCTION}

$\mathbf{M}$ ULTIPATH fading channels encountered in wireless communications afford inherent diversity that can be exploited via appropriate signaling and reception. The key to maximal exploitation of diversity is an understanding of the interaction between the channel and the signal space. In particular, accessing diversity modes at both the transmitter and the receiver greatly facilitates reliable communication over fading channels. Code-division multiple-access (CDMA) receivers exploit multipath diversity via the RAKE structure [1]. Orthogonal frequency-division multiplexing (OFDM) systems

Paper approved by R. Kohno, the Editor for Spread Spectrum Theory and Applications of the IEEE Communications Society. Manuscript received November 25, 2001; revised January 15, 2002 and January 27, 2003. This work was supported in part by the Wisconsin Alumni Research Foundation and in part by the National Science Foundation under Grant CCR-9875805. This paper was presented in part at the IEEE International Symposium on Information Theory, Sorrento, Italy, June 2000, and in part at the 38th Annual Allerton Conference on Communication, Control and Computing, Monticello, IL, October 2000

A. Ganesan is with the Department of Electrical Engineering and Computer Sciences (EECS), University of California-Berkeley, Berkeley, CA 94720 USA (e-mail: ganesan@eecs.berkeley.edu).

A. M. Sayeed is with the Department of Electrical and Computer Engineering, University of Wisconsin-Madison, Madison, WI 53706 USA (e-mail akbar@engr.wisc.edu).

Digital Object Identifier 10.1109/TCOMM.2003.814193 enable diversity exploitation at both the transmitter and receiver by decomposing the channel into parallel noninterfering multicarrier channels. Antenna arrays provide yet another means for diversity in the case of weak coupling between antenna elements.

In this paper, we develop a virtual multiple-input multiple-output (MIMO) framework for single-transmitter, single-receiver multipath fading channels that enables maximal exploitation of channel diversity at both the transmitter and receiver. The essence of the framework is a representation of the system with respect to appropriately chosen sets of basis waveforms for the signal space that expose the inherent structure of the channel. It is assumed throughout that the receiver has perfect channel state information (CSI), while the transmitter has knowledge of only the channel statistics.

The proposed framework has several advantages. First, it provides a general approach to studying the interaction between the signal space and the channel and unifies different existing systems under a common framework. Second, it facilitates design of various signaling and reception schemes (transceiver structures) that enable bandwidth-efficient communication. In particular, it suggests CDMA-based transceiver structures that enable direct application of existing codes developed for OFDM systems and space-time codes developed for multiantenna systems. Finally, it greatly facilitates capacity assessments (both ergodic and outage capacities) for various transceiver structures. In particular, a simple transceiver structure yields simple lower and upper bounds for the capacity of multipath fading channels.

We use the word virtual MIMO to emphasize that the multiple inputs and multiple outputs are created by dimensions of the temporal signaling scheme, not by physical elements such as antennas. Multiantenna systems have received considerable attention recently for supporting very high data rates by employing multiple antennas at both the transmitter and the receiver [2]. Such systems are typically modeled by a Rayleigh fading channel matrix, with entries that are uncorrelated, complex Gaussian random variables of equal variance [3]. When the receiver has perfect CSI and the transmitter is aware of only the channel statistics, space-time codes proposed in [4] take advantage of this simple channel structure. MIMO representations of multipath channels differ from those of multiantenna systems only in the structure and statistics of the channel matrix. Using appropriately chosen MIMO representations, we show that it is possible to expose a structure inherent to the multipath channel which can be exploited at the transmitter in the absence of CSI.

The virtual MIMO framework is also used to obtain capacity expressions for various transceiver structures. The framework reveals clearly the two key parameters that affect the capacity of 
a particular transceiver structure: the number of parallel channels and the level of diversity achieved by the transceiver. Our results show that ergodic capacity is primarily governed by the number of parallel channels and is independent of the level of diversity. On the other hand, outage capacity, a more useful metric in practical systems, is intimately affected by the level of diversity, as well. These results formalize and confirm observations that have been reported in other studies; e.g., see [5] and [6]. Furthermore, new expressions that bound the outage capacity performance of the multipath channel are also obtained.

The rest of the paper is organized as follows. Section II describes the channel model used in the rest of this paper and reviews previous results from the literature. In Section III, we develop virtual MIMO representations of the multipath channel. Section IV describes how the various MIMO representations can be realized in CDMA systems. In Section V, the MIMO framework is used to analyze the ergodic and outage capacity performance of the channel. Section VI contains examples that illustrate different aspects of the proposed framework. Section VII contains concluding remarks.

\section{Channel Model AND PREvious CAPACity Results}

\section{A. Multipath Channel}

We consider spread-spectrum signaling over a slowly fading frequency-selective channel with impulse response $h(\tau)$. The baseband received signal $y(t)$ can be generally expressed as

$$
y(t)=\int_{0}^{T_{m}} h(\tau) x(t-\tau) d \tau+w(t)
$$

where $x(t)$ is the transmitted signal of duration $T$ seconds, twosided bandwidth $B \mathrm{~Hz}$, and average power $P . w(t)$ is additive white Gaussian noise (AWGN) with spectral density $\sigma^{2}$, and $T_{m}$ denotes the multipath spread of the channel. Due to essentially finite signaling bandwidth, $y(t)$ can be accurately approximated as [1], [7]

$$
y(t) \approx \sum_{l=0}^{L-1} h_{l} x\left(t-\frac{l}{B}\right)+w(t)
$$

where $L \doteq\left\lceil T_{m} B\right\rceil$ and $h_{l} \doteq \hat{h}(l / B)$, where $\hat{h}(\tau)$ is a smoothed version of $h(\tau)$ [7]. The number of resolvable components and the number of channel degrees of freedom are both $L$. We consider an uncorrelated scattering, Rayleigh fading channel, for which the delay coefficients $h_{l}$ are uncorrelated, complex zero-mean Gaussian random variables. Average channel power is normalized to unity: $E\left[\sum_{l}\left|h_{l}\right|^{2}\right]=1$. It is noted that the marginal statistics of the frequency response $H(f) \doteq \sum_{l=0}^{L-1} h_{l} e^{-i 2 \pi f l / B}$ are independent of $L$ and $f$; $|H(f)|^{2} \sim \chi^{2}(2)$. However, the correlation between $H(f)$ 's at different frequencies is affected by $L$. It is assumed that the symbol duration $T$ is much larger than the channel delay spread $T_{m}$; i.e., $T \gg T_{m}$, or equivalently, $N \gg L$, so that intersymbol interference can be ignored.

\section{B. Motivation for MIMO Channel Representation}

There are approximately $N=T B$ dimensions in the space of signals with duration $T$ and essential two-sided bandwidth
$B$ [8]. Analogous to [9], our approach to MIMO channel characterization is to represent the channel with respect to a basis for the signal space. The model (2) can then be expressed in the form $\mathbf{y}=\mathbf{H x}+\mathbf{w}$, where $\mathbf{y}, \mathbf{x}$, and $\mathbf{w} \in \mathcal{C}^{N}$ are representations of $y(t), x(t)$, and $w(t)$ with respect to the basis functions, respectively, and $\mathbf{H}$ is the effective channel matrix represented with respect to the basis.

Nyquist sampling ( $B$ samples/second) of the frequency-selective channel (2) leads to the following discrete-time representation of the system:

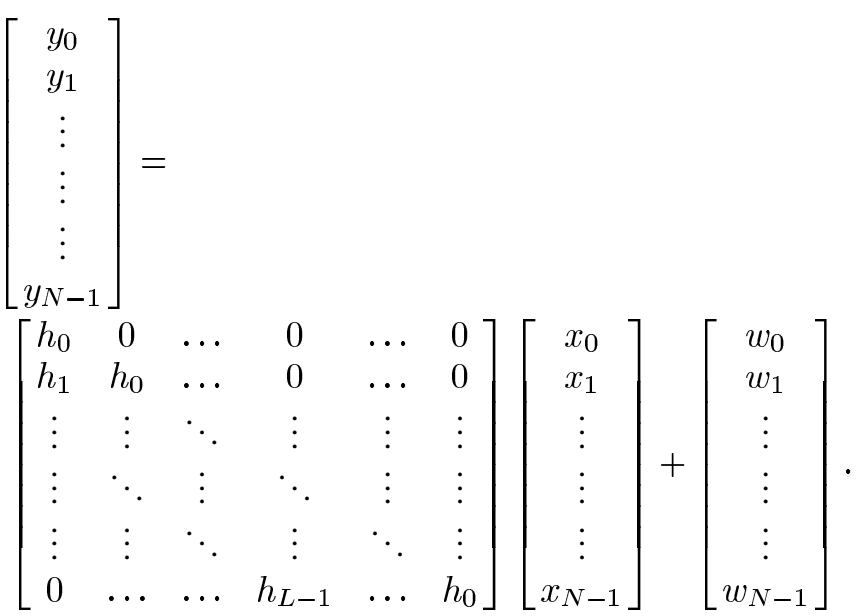

Here, $x_{n}=x(n / B), y_{n}=y(n / B)$, and $w_{n}=w(n / B)$ are time samples of the respective continuous time signals. The channel matrix has a Toeplitz structure, with elements $\left(h_{0}, h_{1}, \ldots, h_{L-1}\right)$ being independent and identically distributed (i.i.d.) complex Gaussian random variables. Particular transceiver structures correspond to transmitting and receiving symbols on a subset of the available dimensions, and can be described by a submatrix equation that is a special case of the channel model. Instead of using the Nyquist sampling basis, a different set of basis functions can be used to characterize the signal space, leading to a different channel representation that is equivalent to the above representation up to linear transformations. In this paper, we show how the choice of basis facilitates analysis and design of novel transceivers.

\section{Notation and Assumptions}

The descriptor $(M, K)$ is used to denote $M$-input $K$-output systems; for example, the system given by (3) is an $(N, N)$ system. All logarithms used in this paper are to base 2 . The capacity achieved by an $(N, N)$ system is expressed in bits. The transmitted signal power is constrained to $P$. It is assumed that AWGN spectral density $\sigma^{2}=1$, noting that, in all subsequent analysis, the only modification necessary for arbitrary value of $\sigma^{2}$ is that $P$ be replaced by $P / \sigma^{2}$. Thus, henceforth, the variable $P$ represents both total transmit power over all $N$ dimensions as well as average received power, due to normalized average channel power.

\section{Ergodic and Outage Capacity}

The two information-theoretic measures we study in this paper are ergodic capacity and outage capacity [6]. The 
ergodic capacity results hold whenever the channel realizations are generated from an ergodic random process and when no decoding delay constraints exist. The transmission sequence can then be made long enough to experience the statistics of the channel. The ergodic capacity is the maximum achievable rate, and is obtained by averaging the mutual information, conditioned on a given channel realization, over the channel statistics [6]. When the signaling duration is finite, capacity becomes a random variable due to the randomness in the channel. For any given rate $R$, there exists a nonzero probability $p$ that the channel realization over this time duration is such that the corresponding channel capacity falls below $R$, and hence, rate $R$ cannot be achieved arbitrarily reliably. Thus, capacity in the strict Shannon sense is zero. It thus makes sense to examine capacity versus outage characteristics. The outage or failure probability for a given rate $R$ is the probability that the capacity, conditioned on the channel realization, falls below $R$. The outage capacity for a given outage or failure probability $p$ is the maximum rate that can be supported by the channel with probability $(1-p)$. In practice, due to finite decoding delay constraints, outage capacity is a more realistic capacity measure.

\section{E. Previous Capacity Results}

The main existing results on the ergodic capacity (denoted by $C_{\text {erg }}$ ) and outage capacity of multipath channels are due to Ozarow [5]. The ergodic capacity can be obtained by starting with (3). Using the fact that for $N \approx B T \gg 1$ the density of eigenvalues of a Toeplitz matrix converges to the power spectrum of $H(f)$ [10], it is shown that the ergodic capacity of the channel over duration $T$, bandwidth $B$, and with power constraint $P$, is given by

$$
C_{\text {erg }}=T E\left[\int_{-B / 2}^{+B / 2} \log \left(1+\frac{P|H(f)|^{2}}{N}\right) d f\right] \text { bits }
$$

where $H(f)$ is assumed to remain constant over the duration of $T$ seconds, and the expectation in (4) is taken over the statistics of $H(f)$. As mentioned earlier, $|H(f)|^{2} \sim \chi^{2}(2)$ for each $f$. Thus, we can write

$$
C_{\text {erg }}=T B E_{\lambda}\left[\log \left(1+\frac{P \lambda}{N}\right)\right]
$$

where $\lambda \sim \chi^{2}(2)$. Now, (5) is also the ergodic capacity of a Rayleigh flat-fading channel (a multipath channel with $L=1$ ) over the same duration, bandwidth, and power constraints. Thus, the ergodic capacity of a multipath channel is independent of $L$, the number of paths. However, this behavior does not hold for outage capacity. Closed-form expressions for outage capacity derived in [5] for $L=1$ and $L=2$ show a significant improvement in performance due to an additional level of diversity. It is conjectured that this improvement will continue as $L$ increases, but closed-form expressions for $L>2$ have not been obtained, due to analytical intractability. Using our framework, we derive new expressions that upper- and lower-bound the outage capacity performance of the multipath channel for arbitrary $L$.

\section{VIRTUAL MIMO REPRESENTATIONS}

In Section III-A, we develop a generic $(N, N)$ matrix representation of the multipath channel in terms of arbitrary basis functions for the signal space. In Section III-B-E, we specialize this result to particular choices of basis functions.

\section{A. MIMO Representation for Arbitrary Basis}

Theorem 1: Suppose $\left\{\phi_{0}(t), \phi_{1}(t), \ldots, \phi_{N-1}(t)\right\} \quad$ is a complete basis for the space of signals with duration $T$ and bandwidth $B$. Suppose $y(t)=\sum_{n=0}^{N-1} y_{n} \phi_{n}(t)$, $x(t)=\sum_{n=0}^{N-1} x_{n} \phi_{n}(t)$, and $w(t)=\sum_{n=0}^{N-1} w_{n} \phi_{n}(t)$. Then, the received signal

$$
y(t)=\sum_{l=0}^{L-1} h_{l} x\left(t-\frac{l}{B}\right)+w(t)
$$

can equivalently be expressed in the form

$$
\mathbf{y}=\mathbf{R}^{-1} \tilde{\mathbf{H}} \mathbf{x}+\mathbf{w}
$$

where $R_{j k} \doteq\left\langle\phi_{k}, \phi_{j}\right\rangle$ and

$$
\tilde{H}_{m n} \doteq \sum_{l=0}^{L-1} h_{l} \int_{0}^{T} \phi_{n}\left(t-\frac{l}{B}\right) \phi_{m}^{*}(t) d t
$$

for $j, k, m, n=0,1, \ldots, N-1$. The noise correlation matrix is $E\left[\mathbf{w w}^{H}\right]=\mathbf{R}$.

Proof: The elements of the correlation matrix of $\mathbf{w}$ are given by

$$
\begin{aligned}
E\left[w_{n} w_{m}^{*}\right] & =E\left[\int_{t} w(t) \phi_{n}^{*}(t) d t \int_{\tau} w^{*}(\tau) \phi_{m}(\tau) d \tau\right] \\
& =\int_{t} \int_{\tau} E\left[w(t) w^{*}(\tau)\right] \phi_{m}(\tau) \phi_{n}^{*}(t) d \tau d t \\
& =\int_{t} \int_{\tau} \delta(t-\tau) \phi_{m}(\tau) \phi_{n}^{*}(t) d \tau d t=R_{m n} .
\end{aligned}
$$

Now, by expressing $x(t)$ and $w(t)$ in terms of basis functions, (6) can be rewritten as

$$
y(t)=\sum_{n=0}^{N-1} x_{n} \sum_{l=0}^{L-1} h_{l} \phi_{n}\left(t-\frac{l}{B}\right)+\sum_{n=0}^{N-1} w_{n} \phi_{n}(t) .
$$

Let $\mathbf{p}$ represent the vector of projections of the received signal onto the basis functions. That is

$$
p_{m} \doteq\left\langle y(t), \phi_{m}(t)\right\rangle=\int_{0}^{T} y(t) \phi_{m}^{*}(t) d t .
$$

for $m=0,1, \ldots, N-1$. Now, substituting (9) into (10) leads to

$$
\sum_{n=0}^{p_{m}=} x_{n} \sum_{l=0}^{L-1} h_{l} \int_{0}^{T} \phi_{n}\left(t-\frac{l}{B}\right) \phi_{m}^{*}(t) d t+\sum_{n=0}^{N-1} w_{n} R_{m n}
$$


with $R_{j k}$ as defined in the Theorem above. Indeed, if we define

$$
\tilde{H}_{m n} \doteq \sum_{l=0}^{L-1} h_{l} \int_{0}^{T} \phi_{n}\left(t-\frac{l}{B}\right) \phi_{m}^{*}(t) d t
$$

the Theorem follows by noting that $\mathbf{p}$ can be expressed as

$$
\mathbf{p}=\tilde{\mathbf{H}} \mathbf{x}+\mathbf{R w} .
$$

In the $(N, N)$ MIMO model $\mathbf{y}=\mathbf{R}^{-1} \tilde{\mathbf{H}} \mathbf{x}+\mathbf{w}, \quad \mathbf{R}^{-1} \tilde{\mathbf{H}}$ can be viewed as the effective channel matrix represented with respect to the basis. As we show later, the basis functions can be chosen so that $\mathbf{R}^{-1} \tilde{\mathbf{H}}$ has a simple structure. The transceivers proposed later effectively exploit this structure at the transmitter in the absence of CSI.

\section{B. Time-Domain Model}

The time-domain representation of the multipath channel is a MIMO representation where the input vector, noise vector, and output vector are time samples of the continuous-time signals $x(t), w(t)$, and $y(t)$, respectively. While such a representation can be obtained as a special case of Theorem 1, it can also be obtained in a straightforward manner from the discrete-time representation given by (3) where the channel matrix is Toeplitz. For large $N$, Toeplitz matrices can be approximated by circular ones [10], [11]. In our case, this is done by replacing the zeros in the upper-right corner of (3) by the delay coefficients $\left\{h_{l}\right\}$ so as to produce a circulant channel matrix. Thus, for $N \approx T B \gg 1$, this results in the time-domain model $\mathbf{y}^{t}=\mathbf{H} \mathbf{x}^{t}+\mathbf{w}^{t}$, as shown in (14) at the bottom of the page, with $\mathbf{w}^{t} \sim N(\mathbf{0}, \mathbf{I})$. The superscript $t$ denotes time-domain representation. Since $\mathbf{H}$ is circulant, it has the eigendecomposition $\mathbf{H}=\mathbf{U} \boldsymbol{\Lambda} \mathbf{U}^{H}$, where $\mathbf{U}^{H}$ is the normalized discrete Fourier transform (DFT) matrix, $\mathbf{U}_{i k}=e^{j 2 \pi i k / N} / \sqrt{N}$ for $i, k=0,1, \ldots, N-1$, and $\mathbf{U}^{H} \mathbf{U}=\mathbf{U U}^{H}=\mathbf{I} . \boldsymbol{\Lambda}=\operatorname{diag}\left(\lambda_{0}, \lambda_{1}, \ldots, \lambda_{N-1}\right)$, where $\lambda_{n}=\sum_{n=0}^{L-1} h_{l} e^{-j 2 \pi n l / N}=\left.H(f)\right|_{f=2 \pi n / N}$ are samples of $H(f)$ that are zero-mean, complex Gaussian random variables with variance one.

Remark: The models we derive next are based on the above circulant approximation of the Toeplitz matrix. The authors are not aware of any theoretical results that quantify this approximation error for finite $N$, and such an analysis is beyond the scope of this paper. However, in Fig. 1, we provide capacity curves of

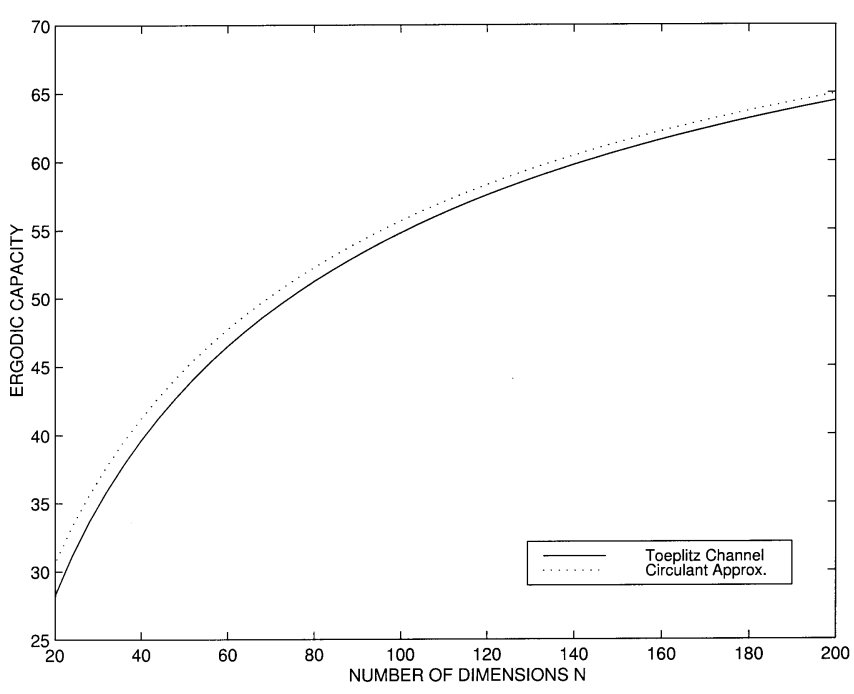

Fig. 1. Toeplitz channel and its circulant approximation.

the Toeplitz channel and its circulant approximation for $L=4$, signal-to-noise ratio (SNR) of $15 \mathrm{~dB}$, and various values of $N$. The simulations, based on 1000 channel realizations, show that the approximation error is negligible for finite, practically large $N$. For example, for $N=128$, the capacities are 59.2 and $58.5 \mathrm{~b}$, respectively. Doing the circulant approximation is equivalent to doing cyclic prefixing, which is done routinely in OFDM systems with a slight loss in rate. As the signaling duration increases (hence, as $N$ increases), this loss becomes smaller. From here on, to facilitate transceiver analysis and design, we use the circulant model, noting that the performance curves we obtain later for transceivers using our framework will be close enough in practice to the true performance of these transceivers. As illustrated later, a novelty of our work lies in being able to apply existing transceiver designs that are based on OFDM and space-time codes to other systems (such as CDMA systems). The above approximation makes this possible.

\section{Frequency-Domain Model}

The frequency-domain samples are obtained from the timedomain samples by taking the DFT. For example, for $x(t)$, we define

$$
\mathbf{x}^{f} \doteq \mathbf{U}^{H} \mathbf{x}^{t}
$$

$$
\left[\begin{array}{c}
y_{0}^{t} \\
y_{1}^{t} \\
\vdots \\
y_{L-2}^{t} \\
y_{L-1}^{t} \\
y_{L}^{t} \\
\vdots \\
y_{N-2}^{t} \\
y_{N-1}^{t}
\end{array}\right]=\left[\begin{array}{cccccccc}
h_{0} & 0 & \ldots & 0 & h_{L-1} & \cdots & h_{2} & h_{1} \\
h_{1} & h_{0} & \ldots & 0 & 0 & \ddots & h_{3} & h_{2} \\
\vdots & \vdots & \ldots & & & & \ddots & \vdots \\
h_{L-2} & h_{L-3} & \cdots & & & & 0 & h_{L-1} \\
h_{L-1} & h_{L-2} & \cdots & & & & 0 & 0 \\
0 & h_{L-1} & \cdots & & & & 0 & 0 \\
\vdots & \vdots & \ddots & \vdots & \vdots & & \vdots & \vdots \\
0 & 0 & & h_{L-2} & h_{L-3} & \ldots & h_{0} & 0 \\
0 & 0 & & h_{L-1} & h_{L-2} & \ldots & h_{1} & h_{0}
\end{array}\right]\left[\begin{array}{c}
x_{0}^{t} \\
x_{1}^{t} \\
\vdots \\
x_{L-2}^{t} \\
x_{L-1}^{t} \\
x_{L}^{t} \\
\vdots \\
x_{N-2}^{t} \\
x_{N-1}^{t}
\end{array}\right]+\left[\begin{array}{c}
w_{0}^{t} \\
w_{1}^{t} \\
\vdots \\
w_{L-2}^{t} \\
w_{L-1}^{t} \\
w_{L}^{t} \\
\vdots \\
w_{N-2}^{t} \\
w_{N-1}^{t}
\end{array}\right]
$$


where the superscript $f$ denotes frequency-domain representation of the signal. This straightforwardly leads to the frequencydomain model $\mathbf{y}^{f}=\Lambda \mathbf{x}^{f}+\mathbf{w}^{f}$

$$
\begin{gathered}
{\left[\begin{array}{c}
y_{0}^{f} \\
y_{1}^{f} \\
\vdots \\
\vdots \\
y_{N-1}^{f}
\end{array}\right]=} \\
{\left[\begin{array}{ccccc}
\lambda_{0} & 0 & \ldots & 0 & 0 \\
0 & \lambda_{1} & \ldots & 0 & 0 \\
\vdots & \vdots & \ddots & \vdots & \vdots \\
\vdots & \vdots & \ldots & \lambda_{N-2} & 0 \\
0 & \ldots & \ldots & 0 & \lambda_{N-1}
\end{array}\right]\left[\begin{array}{c}
x_{0}^{f} \\
x_{1}^{f} \\
\vdots \\
\vdots \\
x_{N-1}^{f}
\end{array}\right]+\left[\begin{array}{c}
w_{0}^{f} \\
w_{1}^{f} \\
\vdots \\
\vdots \\
w_{N-1}^{f}
\end{array}\right]}
\end{gathered}
$$

with $\mathbf{w}^{f} \sim N(0, \mathbf{I})$. This is exactly the model used in OFDM systems. The system consists of $N$ noninterfering parallel channels in the frequency domain, with each parallel channel undergoing Rayleigh fading.

\section{Wideband Circulant CDMA Model}

We consider the special case of Theorem 1 when the basis functions $\left\{\phi_{n}(t)\right\}_{n=1}^{N-1}$ are spread-spectrum waveforms. In a direct-sequence code-division multiple-access (DS-CDMA) system, the information symbol $x_{0}$ is modulated onto a spreading waveform $q_{0}(t)$

$$
q_{0}(t) \doteq \sum_{k=0}^{N-1} c_{k} v\left(t-\frac{k}{B}\right)
$$

and transmitted. Here, $\left\{c_{k}\right\}_{k=0}^{N-1} \in\{+1,-1\}^{N}$ is the spreading code, $v(t)$ is the chip waveform of duration $1 / B$ and approximate bandwidth $B$, and $N$ is the processing gain.

Starting with $q_{0}(t)$, we now construct the basis $\left\{q_{n}(t)\right\}_{n=0}^{N-1}$ as follows. Define

$$
\mathbf{c}^{0} \doteq\left[c_{0}, c_{1}, \ldots, c_{N-1}\right]^{T}
$$

the spreading code of $q_{0}(t)$. Furthermore, for $n=1,2, \ldots, N-$ 1 , define $q_{n}(t)$ to be the $n$th circularly time-shifted version of $q_{0}(t)$. That is

$$
q_{n}(t) \doteq \sum_{k=0}^{N-1} c_{N-n+k} v\left(t-\frac{k}{B}\right)
$$

or equivalently, define $q_{n}(t)$ in terms of its spreading code

$$
\mathbf{c}^{n} \doteq\left[c_{N-n}, c_{N-n+1}, \ldots, c_{N-n-1}\right]^{T} .
$$

For example, $q_{1}(t)$ has the spreading code $\mathbf{c}^{1}=$ $\left[c_{N-1}, c_{0}, c_{1}, \ldots, c_{N-2}\right]$. Now, with a good choice of initial spreading code $\mathbf{c}^{0}$, the waveforms $\left\{q_{n}(t)\right\}_{n=0}^{N-1}$ will be linear independent, and hence, form a complete basis for the signal space. Thus, the transmitted signal $x(t)$ can be expressed in terms of $\left\{q_{n}(t)\right\}_{n=0}^{N-1}$, and the corresponding expanding coefficients $\left\{x_{n}^{c}\right\}_{n=0}^{N-1}$ by

$$
x(t)=\sum_{n=0}^{N-1} x_{n}^{c} q_{n}(t) .
$$

Before proceeding to obtain a MIMO relation for this basis, we note that an important property of this basis is that delayed versions of a spreading waveform can be approximated by circularly time-shifted versions. For example, suppose that the signal $q_{0}(t)$ with spreading code $\left[c_{0}, c_{1}, \ldots, c_{N-1}\right]$ is transmitted. The first delay of $q_{0}(t)$ at the receiver is $q_{0}(t-1 / B)$. This delayed waveform can be represented by the $(N+1)$ dimensional spreading code $\left[0, c_{0}, c_{1}, \ldots, c_{N-2}, c_{N-1}\right]$. The first $N$ dimensions $\left[0, c_{0}, c_{1}, \ldots, c_{N-2}\right]$ are well-approximated by $\left[c_{N-1}, c_{0}, c_{1}, \ldots, c_{N-2}\right]$, which is the spreading code of $q_{1}(t)$. Now, the transmitted signal, after dispersion by the channel, is delayed by at most $L / B$ at the receiver. Thus, for $N \gg L$, a delayed version of a spreading waveform can be approximated by a circularly-shifted version as follows

$$
q_{n}\left(t-\frac{l}{B}\right) \approx q_{[n+l]}(t)
$$

where $[n+l] \doteq(n+l) \bmod N$. A multipath channel produces a linear combination of delayed copies of the transmitted signal at the receiver. For this reason, in the context of CDMA systems, it is natural to use a spreading waveform and circularly timeshifted versions of it as a basis for the signal space. ${ }^{1}$

A direct application of Theorem 1 to basis functions $\left\{q_{n}(t)\right\}$ leads to

$$
\begin{aligned}
\tilde{H}_{m n} & =\sum_{l=0}^{L-1} h_{l} \int_{0}^{T} q_{n}\left(t-\frac{l}{B}\right) q_{m}^{*}(t) d t \\
& =\sum_{l=0}^{L-1} h_{l} \int_{0}^{T} q_{[n+l]}(t) q_{m}^{*}(t) d t \\
& =\sum_{l=0}^{L-1} h_{l} R_{m,[n+l]}
\end{aligned}
$$

where $R_{i j}=\left\langle q_{j}, q_{i}\right\rangle$. From (21), it is evident that $\tilde{\mathbf{H}}$ can be expressed in the form $\tilde{\mathbf{H}}=\mathbf{R H}$, where $\mathbf{H}$ is the same circulant matrix of the time-domain model. To summarize, given

$$
\begin{aligned}
y(t) & =\sum_{n=0}^{N-1} y_{n}^{c} q_{n}(t) \\
x(t) & =\sum_{n=0}^{N-1} x_{n}^{c} q_{n}(t) \\
w(t) & =\sum_{n=0}^{N-1} w_{n}^{c} q_{n}(t)
\end{aligned}
$$

we obtain the wideband circulant CDMA model $\mathbf{y}^{c}=\mathbf{H x}^{c}+\mathbf{w}^{c}$ as shown in (23) at the bottom of the next page, where the superscript $c$ denotes that circulant CDMA waveforms are used as the basis. The noise correlation matrix is given by $E\left[\mathbf{w}^{c}\left(\mathbf{w}^{c}\right)^{H}\right]=\mathbf{R}$.

${ }^{1}$ Under the assumption $T_{m} \ll T$, linear time shifts can be approximated by circular time shifts. In practice, cyclic prefixes can be used to make this approximation exact. Circulant shifts are used for the purpose of exact channel diagonalization. 


\section{E. Wideband Parallel Model}

We obtain another MIMO model by taking the DFT of the wideband CDMA model. For a signal $x(t)$, we define

$$
\mathbf{x}^{p} \doteq \mathbf{U}^{H} \mathbf{x}^{c}
$$

where the superscript $p$ denotes parallel. It follows that

$$
\begin{aligned}
\mathbf{y}^{p} \doteq \mathbf{U}^{H} \mathbf{y}^{c} & =\mathbf{U}^{H}\left(\mathbf{H} \mathbf{x}^{c}+\mathbf{w}^{c}\right) \\
& =\mathbf{U}^{H}\left(\mathbf{U} \boldsymbol{\Lambda} \mathbf{U}^{H} \mathbf{x}^{c}+\mathbf{U} \mathbf{w}^{p}\right)=\mathbf{\Lambda} \mathbf{x}^{p}+\mathbf{w}^{p} .
\end{aligned}
$$

The wideband parallel model is thus given by $\mathbf{y}^{p}=\mathbf{\Lambda} \mathbf{x}^{p}+\mathbf{w}^{p}$

$$
\begin{gathered}
{\left[\begin{array}{c}
y_{0}^{p} \\
y_{1}^{p} \\
\vdots \\
\vdots \\
y_{N-1}^{p}
\end{array}\right]=} \\
{\left[\begin{array}{ccccc}
\lambda_{0} & 0 & \ldots & 0 & 0 \\
0 & \lambda_{1} & \ldots & 0 & 0 \\
\vdots & \vdots & \ddots & \vdots & \vdots \\
\vdots & \vdots & \ldots & \lambda_{N-2} & 0 \\
0 & \ldots & \ldots & 0 & \lambda_{N-1}
\end{array}\right]\left[\begin{array}{c}
x_{0}^{p} \\
x_{1}^{p} \\
\vdots \\
\vdots \\
x_{N-1}^{p}
\end{array}\right]+\left[\begin{array}{c}
w_{0}^{p} \\
w_{1}^{p} \\
\vdots \\
\vdots \\
w_{N-1}^{p}
\end{array}\right]}
\end{gathered}
$$

where $\mathbf{w}^{p} \sim N\left(\mathbf{0}, \mathbf{U}^{H} \mathbf{R U}\right)$.

\section{F. Relation Between Time-Domain and Wideband CDMA \\ Domains}

In this subsection, we consider chip-rate sampling to obtain a relation between signal representations in time-domain and wideband CDMA domain. In the wideband circulant domain, $x(t)$ is represented by $\mathbf{x}^{c}$ and is given by

$$
x(t)=\sum_{n=0}^{N-1} x_{n}^{c} q_{n}(t) .
$$

When each chip waveform has approximate duration $1 / B$, we can perform chip-rate sampling. With $\mathbf{c}^{n}$ denoting the spreading code of $q_{n}(t)$ as before, $x(t)$ can be rewritten in the form

$$
\mathbf{x}^{t}=\sum_{n=0}^{N-1} x_{n}^{c} \mathbf{c}^{n} .
$$

Define the matrix of spreading codes $\mathbf{C} \doteq\left[\mathbf{c}^{0}, \mathbf{c}^{1}, \ldots, \mathbf{c}^{N-1}\right]$. Then

$$
\mathbf{x}^{t}=\mathbf{C} \mathbf{x}^{c} .
$$

Equation (27) describes how the representations of $x(t)$ in the time-domain model and wideband CDMA model are related. Similar relations hold, mutatis mutandis, for $y(t)$ and $w(t)$ as well. Using (23) and (27), we obtain

$$
\mathbf{y}^{t}=\mathbf{C} \mathbf{y}^{c}=\mathbf{C}\left(\mathbf{H x}^{c}+\mathbf{w}^{c}\right)=\mathbf{C H C} \mathbf{C}^{-1} \mathbf{x}^{t}+\mathbf{w}^{t} .
$$

Since $q_{n}(t)$ is a circularly time-shifted version of $q_{0}(t), \mathbf{C}$ is a circulant matrix and can be diagonalized by $\mathbf{U}: \mathbf{D} \doteq \mathbf{U}^{H} \mathbf{C U}$ is a diagonal matrix. Since $\mathbf{H}$ is also circulant, the eigenfunctions of $\mathbf{C}$ and $\mathbf{H}$ commute, so that $\mathbf{C H C}^{-1}=\mathbf{H}$. Also, $E\left[\mathbf{w}^{t}\left(\mathbf{w}^{t}\right)^{H}\right]=\mathbf{I}$. Thus, the equation above reduces exactly to the time-domain model derived earlier. While the time-domain model derived this way does not produce any new model, it is presented because chip-rate sampling is used in practice. We have essentially shown that the relation between $\mathbf{x}^{t}$ and $\mathbf{x}^{c}$ developed here in the context of chip-rate sampling is consistent with the signal space characterizations developed earlier.

\section{G. Summary}

To summarize, the MIMO channel representations in the four domains are as follows:

$$
\begin{aligned}
\mathbf{y}^{c} & =\mathbf{H} \mathbf{x}^{c}+\mathbf{w}^{c}=\mathbf{U} \mathbf{\Lambda} \mathbf{U}^{H} \mathbf{x}^{c}+\mathbf{w}^{c} \\
\mathbf{w}^{c} & \sim N\left(\mathbf{0},\left(\mathbf{C}^{H} \mathbf{C}\right)\right) . \\
\mathbf{y}^{p} & =\mathbf{\Lambda} \mathbf{x}^{p}+\mathbf{w}^{p}, \quad \mathbf{w}^{p} \sim N\left(\mathbf{0},\left(\mathbf{D}^{H} \mathbf{D}\right)\right) . \\
\mathbf{y}^{t} & =\mathbf{H} \mathbf{x}^{t}+\mathbf{w}^{t}=\mathbf{U} \mathbf{\Lambda} \mathbf{U}^{H} \mathbf{x}^{t}+\mathbf{w}^{t} \\
\mathbf{w}^{t} & \sim N(\mathbf{0}, \mathbf{I}) . \\
\mathbf{y}^{f} & =\mathbf{\Lambda} \mathbf{x}^{f}+\mathbf{w}^{f}, \quad \mathbf{w}^{f} \sim N(\mathbf{0}, \mathbf{I}) .
\end{aligned}
$$

The four representations of a signal are related as follows:

$$
\begin{aligned}
\mathbf{x}^{f} & =\mathbf{U}^{H} \mathbf{x}^{t} \longleftrightarrow \mathbf{x}^{t}=\mathbf{U} \mathbf{x}^{f} \\
\mathbf{x}^{t} & =\mathbf{C} \mathbf{x}^{c}=\left(\mathbf{U D} \mathbf{U}^{H}\right) \mathbf{x}^{c} \longleftrightarrow \mathbf{x}^{c} \\
& =\left(\mathbf{C}^{H} \mathbf{C}\right)^{-1} \mathbf{C}^{H} \mathbf{x}^{t}=\mathbf{C}^{-1} \mathbf{x}^{t} \\
\mathbf{x}^{p} & =\mathbf{U}^{H} \mathbf{x}^{c} \longleftrightarrow \mathbf{x}^{c}=\mathbf{U} \mathbf{x}^{p} .
\end{aligned}
$$

$$
\left[\begin{array}{c}
y_{0}^{c} \\
y_{1}^{c} \\
\vdots \\
y_{L-2}^{c} \\
y_{L-1}^{c} \\
y_{L}^{c} \\
\vdots \\
y_{N-2}^{c} \\
y_{N-1}^{c}
\end{array}\right]=\left[\begin{array}{cccccccc}
h_{0} & 0 & \ldots & 0 & h_{L-1} & \ldots & h_{2} & h_{1} \\
h_{1} & h_{0} & \ldots & 0 & 0 & \ddots & h_{3} & h_{2} \\
\vdots & \vdots & \ldots & & & & \ddots & \vdots \\
h_{L-2} & h_{L-3} & \ldots & & & & 0 & h_{L-1} \\
h_{L-1} & h_{L-2} & \ldots & & & & 0 & 0 \\
0 & h_{L-1} & \ldots & & & & 0 & 0 \\
\vdots & \vdots & \ddots & \vdots & \vdots & & \vdots & \vdots \\
0 & 0 & & h_{L-2} & h_{L-3} & \ldots & h_{0} & 0 \\
0 & 0 & & h_{L-1} & h_{L-2} & \ldots & h_{1} & h_{0}
\end{array}\right]\left[\begin{array}{c}
x_{0}^{c} \\
x_{1}^{c} \\
\vdots \\
x_{L-2}^{c} \\
x_{L-1}^{c} \\
x_{L}^{c} \\
\vdots \\
x_{N-2}^{c} \\
x_{N-1}^{c}
\end{array}\right]+\left[\begin{array}{c}
w_{0}^{c} \\
w_{1}^{c} \\
\vdots \\
w_{L-2}^{c} \\
w_{L-1}^{c} \\
w_{L}^{c} \\
\vdots \\
w_{N-2}^{c} \\
w_{N-1}^{c}
\end{array}\right]
$$




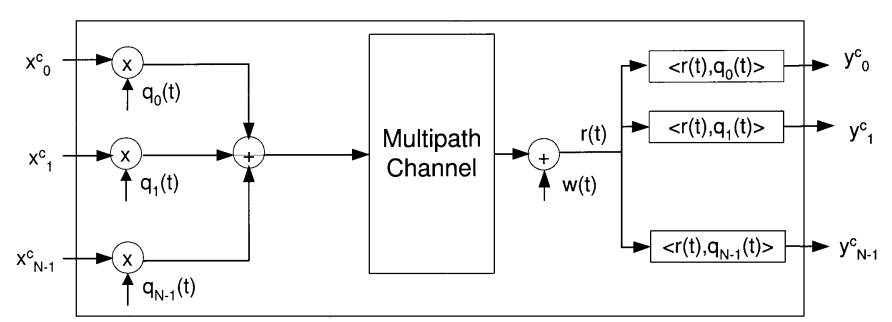

Fig. 2. Wideband CDMA model $\mathbf{y}^{c}=\mathbf{H} \mathbf{x}^{c}+\mathbf{w}^{c}$.

The four MIMO models are all equivalent, up to linear transformations. While any of the four models can be used in subsequent analysis, we use those that make the analysis most tractable. For ergodic capacity related analysis, the parallel and frequency-domain models are the most convenient. For outage capacity analysis, the time-domain and wideband CDMA models provide the most insight. CDMA-based transceivers are naturally best represented in the wideband CDMA domain, while the performance analysis is typically carried out by transforming into the wideband parallel domain.

\section{REALIZING DifFERENT REPRESENTATIONS IN A CDMA SYSTEM}

\section{A. Wideband CDMA System}

We now describe preprocessing and postprocessing that facilitates application of space-time codes to circulant CDMA systems. Consider a CDMA system operating over a multipath channel. The information symbols are modulated onto circularly-shifted copies of a particular spreading waveform $q_{0}(t)$, as illustrated in Fig. 2. The received signal is projected onto the same circularly shifted waveforms to capture the sufficient statistics. The system in Fig. 2 is equivalent to the wideband CDMA model (23). Other researchers have investigated using multiple time-shifted copies of a spreading code for a particular user. In [12], for example, each user is assigned a spreading waveform and a delayed version of it. In contrast, the wideband CDMA system assigns circularly shifted copies of a spreading waveform to each user. This is possible both in systems that employ short codes and in systems that employ long codes.

\section{B. Realizing a Time-Domain System}

Fig. 3 details the preprocessing at the transmitter and postprocessing at the receiver that transform the circulant CDMA system, as depicted in Fig. 2, into a time-domain system. This transforms a CDMA system using circular codes into a Nyquist sampling system. The effective system, $\mathbf{y}^{t}=\mathbf{H} \mathbf{x}^{t}+\mathbf{w}^{t}$, has noise vector that is white. The effective channel matrix, $\mathbf{H}$, is still circulant; it does not contain all uncorrelated elements. However, as we show in a later example (Section VI-D), by transmitting and receiving on a particular subset of the available dimensions, it is possible to create a subsystem whose effective channel matrix contains all uncorrelated elements. Space-time codes can then be applied. See Section VI-D for further details.

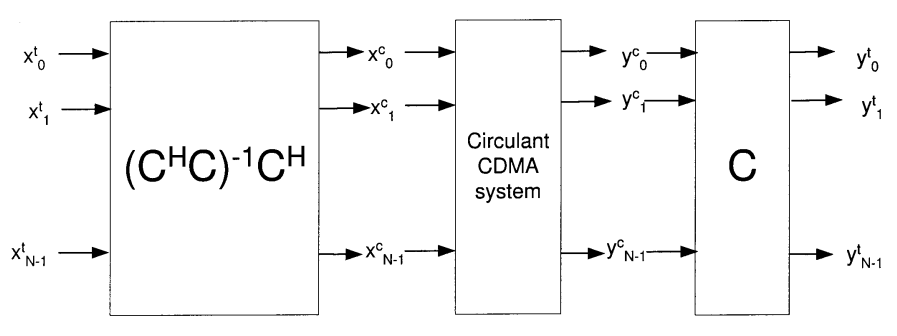

Fig. 3. Realizing the time-domain system $\mathbf{y}^{t}=\mathbf{H} \mathbf{x}^{t}+\mathbf{w}^{t}$ via the wideband CDMA representation.

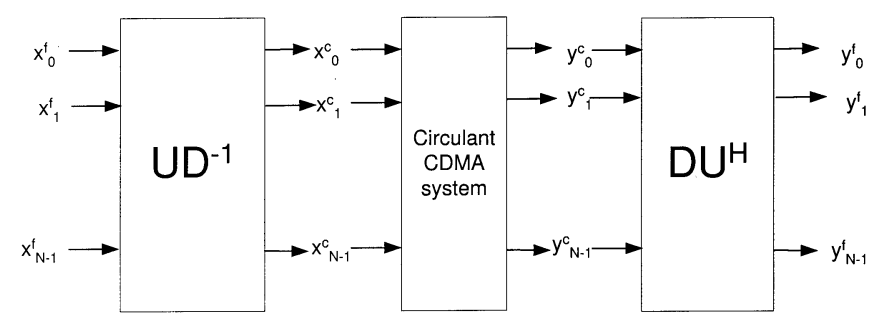

Fig. 4. Realizing the frequency-domain system $\mathbf{y}^{f}=\Lambda \mathbf{x}^{f}+\mathbf{w}^{f}$ via the wideband CDMA representation.

\section{Realizing a Frequency-Domain System}

Fig. 4 details the preprocessing and postprocessing that transform the circulant CDMA system, as depicted in Fig. 2, into a frequency-domain system. OFDM-based codes can now be applied to CDMA systems via appropriate transformations. It is important to keepin mind that we are able to do the preprocessing at the transmittereven without knowledge of the channelcoefficients because the eigenfunctions of the channel are fixed a priori. The multipath channel, by virtue of the delays it produces, has an inherent circulant structure. By transmitting on circularly shifted waveforms that serve as a basis, this structure can be exploited at the transmitter, even in the absence of CSI. The key point is that both $\mathbf{H}$ and $\mathbf{C}$ are circulant. In the transceiver example mentioned above, working with a particular subset of the available dimensions exposes a structure in the channel matrix similar to that found in multiple antenna systems.

\section{CAPACITY}

\section{A. Ergodic Capacity}

We want to determine the maximum average mutual information between the input and output of the multipath channel. We take an approach similar to [3]. While the results in [3] are developed for the channel matrix containing i.i.d. entries, we develop our results for the channel matrix induced by the multipath channel. We start with the frequency-domain model. Once again, we assume the receiver has perfect CSI, while the transmitter has knowledge of only channel statistics. The overall transmitted power is conserved to $P ; E\left[\mathbf{x}^{H} \mathbf{x}\right] \leq P$. The average mutual information is given by

$$
\mathcal{I}\left(\mathbf{x}^{f}, \mathbf{y}^{f}\right)=E_{\boldsymbol{\Lambda}}\left(\mathcal{I}\left(\mathbf{x}^{f}, \mathbf{y}^{f} \mid \mathbf{\Lambda}\right)\right) .
$$

We first determine the maximum mutual information conditioned on a fixed realization $\boldsymbol{\Lambda}$. Then

$$
\mathcal{I}\left(\mathbf{x}^{f}, \mathbf{y}^{f} \mid \boldsymbol{\Lambda}\right)=\mathcal{H}\left(\mathbf{y}^{f}\right)-\mathcal{H}\left(\mathbf{y}^{f} \mid \mathbf{x}^{f}\right)=\mathcal{H}\left(\mathbf{y}^{f}\right)-\mathcal{H}\left(\mathbf{w}^{f}\right) .
$$


The entropy $\mathcal{H}\left(\mathbf{y}^{f}\right)$ is maximized when $\mathbf{y}^{f}$ is circularly symmetric complex Gaussian, which is the case when $\mathrm{x}^{f}$ is also circularly symmetric complex Gaussian [3]. This implies

$$
\begin{aligned}
\mathcal{I}\left(\mathbf{x}^{f}, \mathbf{y}^{f} \mid \mathbf{\Lambda}\right) & =\log \left(\operatorname{det}\left(\pi e \mathbf{Q}_{y}\right)\right)-\log \left(\operatorname{det}\left(\pi e \mathbf{I}_{N}\right)\right) \\
& =\log \left(\operatorname{det}\left(\mathbf{I}_{N}+\mathbf{\Lambda} \mathbf{Q}_{x} \mathbf{\Lambda}^{H}\right)\right)
\end{aligned}
$$

where $\mathbf{Q}_{y}=\boldsymbol{\Lambda} \mathbf{Q}_{x} \boldsymbol{\Lambda}^{H}+\mathbf{I}_{N}$ is the covariance matrix of $\mathbf{y}^{f}$ and $\mathbf{Q}_{x}$ is the covariance matrix of $\mathbf{x}^{f}$. We need to maximize

$$
\mathcal{I}\left(\mathbf{x}^{f}, \mathbf{y}^{f} \mid \mathbf{\Lambda}\right)=\log \left(\operatorname{det}\left(\mathbf{I}_{N}+\mathbf{Q}_{x} \mathbf{\Lambda}^{H} \boldsymbol{\Lambda}\right)\right)
$$

subject to the constraint $\operatorname{tr}\left(\mathbf{Q}_{x}\right)=P$. By Hadamard's inequality [13]

$$
\mathcal{I}\left(\mathbf{x}^{f}, \mathbf{y}^{f} \mid \mathbf{\Lambda}\right) \leq \sum_{n=0}^{N-1} \log \left(1+\left(\mathbf{Q}_{x}\right)_{n n}\left|\lambda_{n}\right|^{2}\right)
$$

with equality if and only if $\mathbf{Q}_{x}$ is diagonal. Thus, we can restrict our attention to diagonal $\mathbf{Q}_{x}$. The ergodic capacity for a given diagonal $\mathbf{Q}_{x}$ is the mutual information averaged over the distribution of $\left\{\lambda_{n}\right\}$

$$
\mathcal{I}\left(\mathbf{x}^{f}, \mathbf{y}^{f}\right)=E_{\boldsymbol{\Lambda}}\left[\sum_{n=0}^{N-1} \log \left(1+\left(\mathbf{Q}_{x}\right)_{n n}\left|\lambda_{n}\right|^{2}\right)\right] .
$$

We now determine the optimal power allocation $\left(\mathbf{Q}_{x}\right)_{n n}$ at the transmitter that maximizes (36). As the transmitter does not know the particular realization of $\boldsymbol{\Lambda}$, water filling based on knowledge of $\lambda_{n}$ cannot be done. Optimizing based on just the channel statistics leads to the following result.

Lemma: The average mutual information $\mathcal{I}(\mathbf{x}, \mathbf{y})=$ $E_{\boldsymbol{\Lambda}}\left[\sum_{n=0}^{N-1} \log \left(1+\left(\mathbf{Q}_{x}\right)_{n n}\left|\lambda_{n}\right|^{2}\right)\right]$, subject to constraint $\operatorname{tr}\left(\mathbf{Q}_{x}\right)=P$, is maximized when $\left(\mathbf{Q}_{x}\right)_{n n}=P / N$ for all $n$.

Proof: Define $\mathbf{P}=\left[P_{0}, P_{1}, \ldots, P_{N-1}\right]^{T}$, the power allocation vector. We want to determine the $\mathbf{P}$ that maximizes

$$
\begin{aligned}
f(\mathbf{P}) & =E_{\boldsymbol{\Lambda}}\left[\sum_{n=0}^{N-1} \log \left(1+P_{n}\left|\lambda_{n}\right|^{2}\right)\right] \\
& =\sum_{n=0}^{N-1} E_{\lambda_{n}}\left[\log \left(1+P_{n}\left|\lambda_{n}\right|^{2}\right)\right]
\end{aligned}
$$

subject to the power constraint $\sum_{n} P_{n}=P$. Given any arbitrary $\mathbf{P}$, define $\mathbf{P}^{i j}$ to be the vector with $P_{i}$ and $P_{j}$ interchanged. For example, $\mathbf{P}^{01}=\left[P_{1}, P_{0}, P_{2}, \ldots, P_{N-1}\right]^{T}$. Then

$$
\begin{aligned}
f\left(\mathbf{P}^{i j}\right)= & E_{\boldsymbol{\Lambda}}\left[\sum_{n \neq i, j} \log \left(1+P_{n}\left|\lambda_{n}\right|^{2}\right)+\log \left(1+P_{i}\left|\lambda_{j}\right|^{2}\right)\right. \\
& \left.\quad+\log \left(1+P_{j}\left|\lambda_{i}\right|^{2}\right)\right] \\
= & \sum_{n \neq i, j} E_{\lambda_{n}} \log \left(1+P_{n}\left|\lambda_{n}\right|^{2}\right) \\
& +E_{\lambda_{j}} \log \left(1+P_{i}\left|\lambda_{j}\right|^{2}\right)+E_{\lambda_{i}} \log \left(1+P_{j}\left|\lambda_{i}\right|^{2}\right) .
\end{aligned}
$$

Since $\left|\lambda_{n}\right|^{2} \sim \chi^{2}(2)$, with distribution independent of $n$

$$
E_{\lambda_{j}} \log \left(1+P_{i}\left|\lambda_{j}\right|^{2}\right)=E_{\lambda_{i}} \log \left(1+P_{i}\left|\lambda_{i}\right|^{2}\right)
$$

and

$$
f\left(\mathbf{P}^{i j}\right)=\sum_{n} E_{\lambda_{n}} \log \left(1+P_{n}\left|\lambda_{n}\right|^{2}\right)=f(\mathbf{P}) .
$$

Let $\mathbf{P}_{0}=(1 / N !) \sum_{i j} \mathbf{P}^{i j}=(P / N)[1,1, \ldots, 1]^{T}$ be the uniform power-allocated scheme that is also the average of all permutations of $\mathbf{P}$. By the concavity of $f(\mathbf{P})$

$$
f\left(\mathbf{P}_{0}\right) \geq \frac{1}{N !} \sum_{i j} f\left(\mathbf{P}^{i j}\right)=f(\mathbf{P}) .
$$

Thus, the mutual information for any arbitrary power-allocation scheme $\mathbf{P}$ is upper bounded by that of the equal power-allocation scheme $\mathbf{P}_{0}$. Hence, the average mutual information is maximum for the uniform power-allocation scheme.

We summarize our result on ergodic capacity in the following theorem.

Theorem: Consider an $L$-path Rayleigh fading channel with $E\left[\sum_{l=0}^{L-1}\left|h_{l}\right|^{2}\right]=1$. Consider the space of signals with duration $T$ and bandwidth $B$, whose dimension is $N \approx T B$. Let the total transmit power be constrained to $P$. The ergodic capacity of the channel in the signal space is given by

$$
\begin{aligned}
C & =E_{\Lambda}\left[\sum_{n=0}^{N-1} \log \left(1+\frac{P}{N}\left|\lambda_{n}\right|^{2}\right)\right] \\
& =N E_{\gamma}\left[\log \left(1+\frac{P}{N} \gamma\right)\right] \text { bits }
\end{aligned}
$$

where $\gamma \sim \chi^{2}(2)$. This is also the ergodic capacity of $N$ parallel scalar Rayleigh fading channels each with average received power $P / N$.

The above result decomposes the frequency-selective channel into $N$ parallel, scalar fading channels. The ergodic capacity (38) is exactly equal to (5), the ergodic capacity of a flat-fading channel with the same power and signal space constraints. In general, for given power and signal-space dimensions, the ergodic capacity depends only on the marginal statistics of $H(f)$, which are independent of $L$ and the power in each multipath component. On the other hand, the outage capacity depends on the correlation between $H(f)$ at various frequencies and is, thus, affected by $L$. For example, for SNR $=15 \mathrm{~dB}$ and $N=64$, Fig. 5 shows the outage capacity curves for $L=1$ to 5 and the ergodic capacity curve. The ergodic capacity is $32.7 \mathrm{~b}$ and is depicted by the vertical line. The curves, obtained numerically from 5000 channel realizations, illustrate the improvement in outage capacity performance with increasing diversity. While these are known results (see [6], for example), our method of derivation is different, and more importantly, the outage-capacity expressions we derive next are new.

\section{B. Outage Capacity}

Outage-capacity analysis of multipath channels has been investigated to some extent in [5]. For example, outage-capacity 


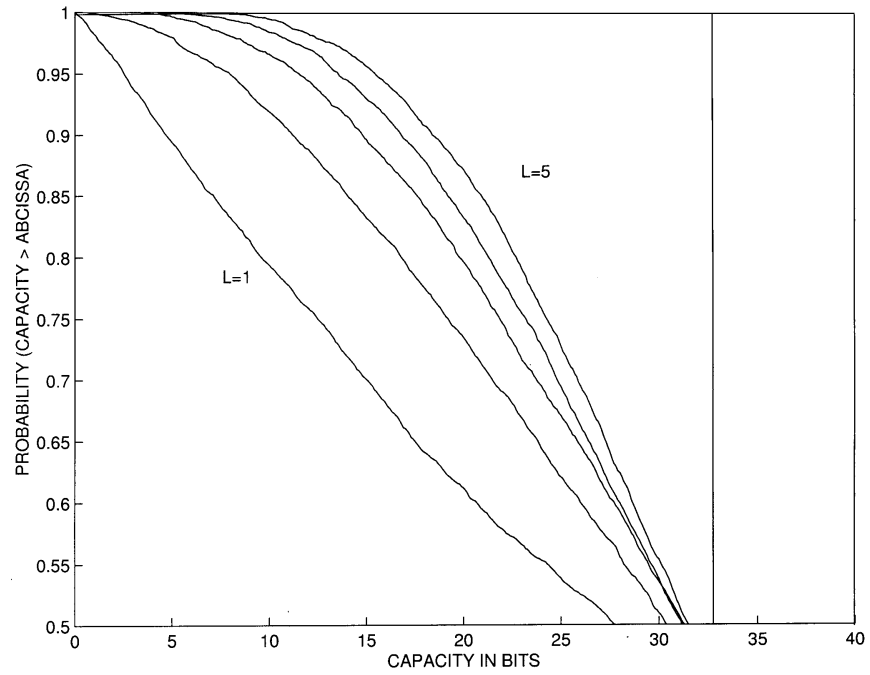

Fig. 5. Outage capacity for $L=1-5$, and ergodic capacity (vertical line).

expressions for $L=2$ are available in [5], whereas expressions for $L>2$ are unavailable due to analytical intractability. In Section VI, we derive simple expressions for approximating the outage capacity that are a function of $P, N$, and $L$. We show that the outage or failure probability for a given rate $R$ is bounded as

$$
\begin{aligned}
\frac{\gamma\left(L, \frac{N}{P}\left(2^{R L / N}-1\right)\right)}{\Gamma(L)} & \leq \operatorname{Pr}(C<R) \\
& \leq \frac{\gamma\left(L, \frac{N L}{P}\left(2^{R / N}-1\right)\right)}{\Gamma(L)}
\end{aligned}
$$

where $\gamma(a, x)$ is the incomplete gamma function $\int_{0}^{x} u^{a-1} e^{-u} d u$. These bounds become tighter as $N$ increases. See Section VI-C for details.

\section{ILLUSTRATIVE EXAMPLES}

In this section, we describe a variety of signaling and reception schemes that illustrate the application of our framework. In particular, we propose two transceiver structures for multipath channels, to which existing codes developed for OFDM and multiantenna systems can be directly applied. For each scheme, the transmitted signal occupies a subset $M$, and the received signal occupies a subset $K$ of $N$ available dimensions. A $(M, K)$ MIMO matrix representation can be used to describe the transceiver to assess performance tradeoffs.

The various transceivers proposed in this section differ in one of four ways: 1) the number of dimensions occupied by the signaling scheme; 2) the number of parallel channels created by the scheme; 3 ) the diversity order attained by the scheme; and 4) received power. The number of parallel channels in an $(M, K)$ system is upper bounded by $\min (M, K)$. The diversity order provided by a scheme corresponds to the number of independently faded replicas of transmitted signal available at the receiver. The higher the diversity order of a scheme, the more the statistical stability, and the steeper the outage capacity curves. The examples to follow help develop an understanding of the tradeoffs between various transceiver structures and provide insights on how each parameter affects the system performance. Most of the transceivers we describe are designed using the

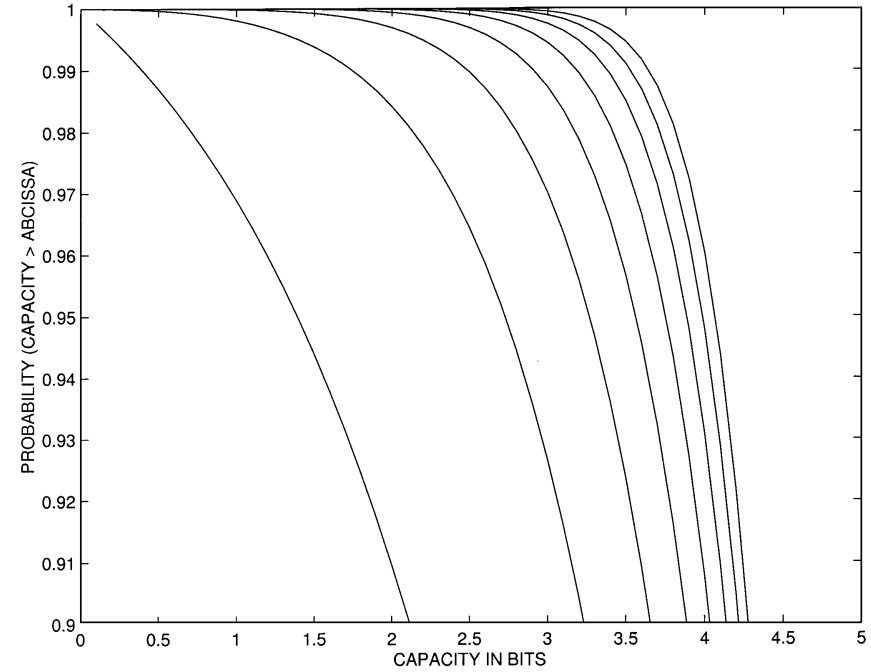

Fig. 6. Outage capacity of $L$ th-order diversity system for $L=1-8$.

time-domain representation for simplicity of exposition. However, these transceivers can be applied to CDMA systems as well, via the transformations detailed in Section IV.

\section{A. RAKE Transceiver-Time-Domain}

This example describes the familiar RAKE transceiver in our framework. Consider transmitting the signal in the time-domain model in just the first time sample. In that case, $\mathbf{x}^{t}=\left[x_{0}, 0, \ldots, 0\right]$ in (14). The receiver needs to probe just the first $L$ time samples, $\left(y_{0}, y_{1}, \ldots, y_{L-1}\right)$, as they contain all the information about the transmitted signal. This transceiver is a $(1, L)$ system

$$
\left[\begin{array}{c}
y_{0} \\
y_{1} \\
\vdots \\
y_{L-1}
\end{array}\right]=\left[\begin{array}{c}
h_{0} \\
h_{1} \\
\vdots \\
h_{L-1}
\end{array}\right] x_{0}+\left[\begin{array}{c}
w_{0} \\
w_{1} \\
\vdots \\
w_{L-1}
\end{array}\right] .
$$

The number of dimensions occupied by the received signal is $L$. The average received power is $P$. This transceiver creates one parallel channel, with gain $\sum_{l=0}^{L-1}\left|h_{l}\right|^{2}$.

The capacity of this subchannel conditioned on a particular channel realization $\left\{h_{l}\right\}$ works out to

$$
C_{1}=\log \left(1+P \sum_{l=0}^{L-1}\left|h_{l}\right|^{2}\right) .
$$

The ergodic capacity of the transceiver is the capacity $C_{1}$ averaged over the channel statistics. For $P=15 \mathrm{~dB}$ and $N=L=$ 4 , the ergodic capacity is $4.5 \mathrm{~b}$. The outage or failure probability is equal to

$$
\begin{aligned}
& \operatorname{Pr}\left(\log \left(1+P \sum_{l=0}^{L-1}\left|h_{l}\right|^{2}\right)<R\right) \\
& =\operatorname{Pr}\left(\sum_{l=0}^{L-1}\left|h_{l}\right|^{2}<\frac{2^{R}-1}{P}\right) \\
& =\frac{\gamma\left(L, \frac{2^{R}-1}{L}\right)}{\Gamma(L)} .
\end{aligned}
$$


A plot of (41) for $L=1,2, \ldots, 8$ is given in Fig. 6. As $L$ increases, the performance improves due to statistical stability of received SNR provided by independent fading. As $L \rightarrow \infty$ (and therefore, $N \rightarrow \infty$ ), the performance of the multipath channel approaches that of a scalar AWGN channel with same average received power. The AWGN channel capacity in this case is $\log (1+P)=5.0 \mathrm{~b}$. Hence, as $L \rightarrow \infty$, the outage capacity curves will approach a steep drop at this value.

\section{B. RAKE Transceiver-CDMA Systems}

This example discusses the RAKE receiver in the context of CDMA systems using the wideband circulant CDMA model. Consider the case where a symbol $x_{0}$ is modulated onto just one spreading waveform $q_{0}(t)$ and transmitted. We have that $\mathbf{x}^{c}=[1,0, \ldots, 0]^{T} x_{0}$. With $\mathbf{c}^{0}$ denoting the spreading code of $q_{0}(t)$, we have that $\mathbf{y}^{c}=\mathbf{H c}^{0} x_{0}+\mathbf{w}^{c}$. The capacity for a particular channel realization $\mathbf{H}=\mathbf{U} \mathbf{\Lambda} \mathbf{U}^{H}$ is given by

$$
C_{2}=\log \left(1+P\left(\mathbf{c}^{0}\right)^{H} \mathbf{H}^{H} \mathbf{H} \mathbf{c}^{0}\right) .
$$

The capacity analysis can be taken one step further by using our framework to transform into the wideband parallel domain, and we get

$$
\mathbf{y}_{p}=\mathbf{D}^{H} \boldsymbol{\Lambda} \mathbf{D} \mathbf{x}^{p}+\mathbf{w}^{p}, \mathbf{w}^{p} \sim N\left(\mathbf{0}, \mathbf{D}^{H} \mathbf{D}\right)
$$

where $\mathbf{x}^{p}=\mathbf{U}^{H} \mathbf{X}^{c}=\mathbf{u}_{0}^{*} x_{0}, \mathbf{u}_{0}$ is the first column of $\mathbf{U}$, and $\mathbf{C}=\mathbf{U D U}^{H}$. Thus

$$
\begin{aligned}
C_{2} & =\log \left(\operatorname{det}\left(1+P \mathbf{u}_{0}^{H} \mathbf{\Lambda}^{H} \mathbf{D}^{H} \mathbf{D} \mathbf{\Lambda} \mathbf{u}_{0}\right)\right) \\
& =\log \left(1+\frac{P}{N} \sum_{n=0}^{N-1}\left|\lambda_{n}\right|^{2}\left|\mathbf{D}_{n n}\right|^{2}\right) .
\end{aligned}
$$

\section{Bounds on the Outage Capacity of the Multipath Channel}

In this example, we provide a simple transceiver whose outage capacity performance approximates that of the multipath channel, and the approximation improves as the dimensionality of the signal space increases. Consider the following transmission strategy in the time-domain model. The signal is transmitted in $M=\lfloor N / L\rfloor$ dimensions, each separated by exactly $L$ time samples. In $(14), \mathbf{x}^{t}=\left[x_{0}, x_{1}, \ldots, x_{L}, x_{L+1}, \ldots, x_{M L}, x_{M L+1}, \ldots\right]^{T}$ $\left[s_{1}, 0, \ldots, s_{2}, 0, \ldots, s_{M}, 0, \ldots\right]^{T}$. Each transmitted signal has average power $P / M$ and encounters $L$ th order diversity. The receiver processes all $N$ dimensions. This is a $(M, N)$ system with $M$ parallel channels. The $M$ parallel channels do not interfere as the transmitted signals are separated by $L$ time samples. The system can be viewed as comprising $M$ parallel RAKE transceivers, and thus, the capacity expressions are easier to compute.

For noninterfering parallel channels, the capacity is simply the sum of the capacity of each parallel channel, which is simply (40) with $P$ replaced by $P / M$. Thus

$$
C_{3}=E_{\mathbf{H}}\left[M \log \left(1+\frac{P \sum_{l=0}^{L-1}\left|h_{l}\right|^{2}}{M}\right)\right] .
$$

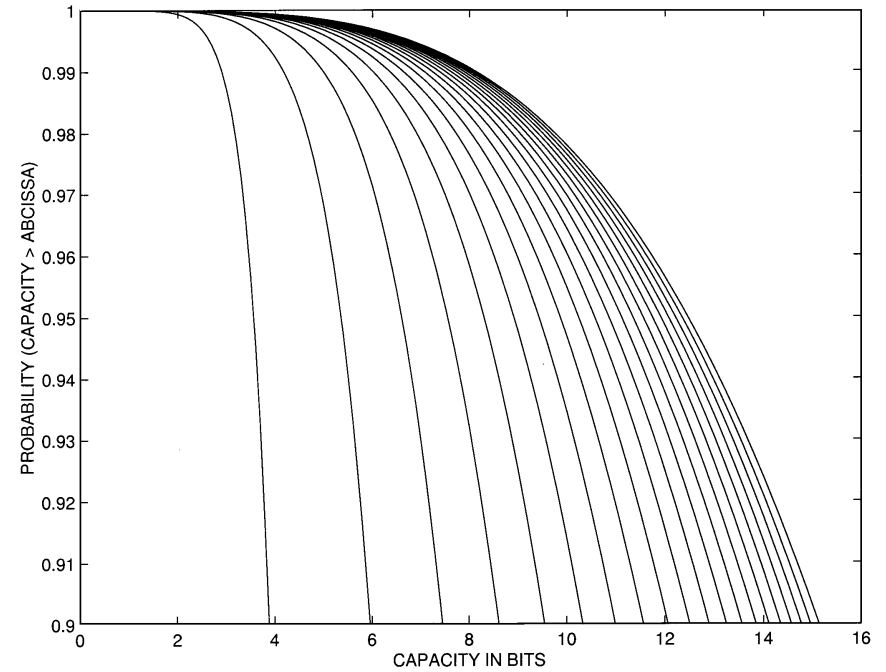

Fig. 7. Outage capacity for $L=4$ and $M=1-20 ; P=15 \mathrm{~dB}$.

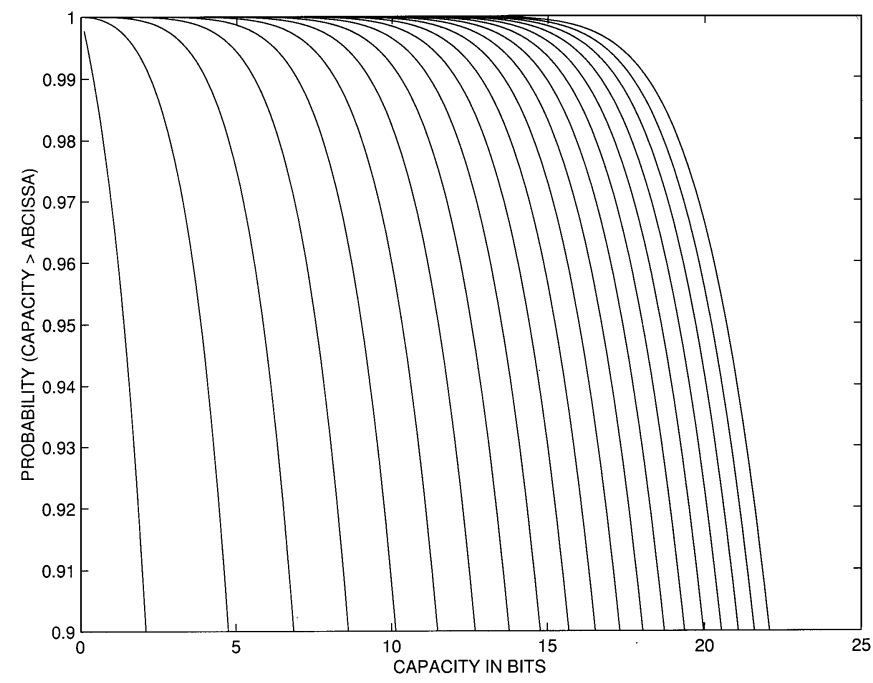

Fig. 8. Outage capacity for $M=L=1-20 ; P=15 \mathrm{~dB}$.

The outage probability for a given rate $R$ equals

$$
\begin{aligned}
& \operatorname{Pr}\left(M \log \left(1+\frac{P}{M} \sum_{l=0}^{L-1}\left|h_{l}\right|^{2}\right)<R\right) \\
& \quad=\operatorname{Pr}\left(\sum_{l=0}^{L-1}\left|h_{l}\right|^{2}<\frac{M}{P}\left(2^{R / M}-1\right)\right) \\
& \quad=\frac{\gamma\left(L, \frac{L M}{P}\left(2^{R / M}-1\right)\right)}{\Gamma(L)} .
\end{aligned}
$$

Fig. 7 shows the outage capacity curves for $L=4, P=15 \mathrm{~dB}$, and $M=1,2, \ldots, 20$. The special case $M=1$ corresponds to the RAKE transceiver of the previous example, with $L=4$. Notice that compared to Fig. 6, the capacity in this scheme takes on larger values due to an increase in the number of parallel channels $M$. However, the curves do not become steeper, since $L$ is kept constant, and hence, there is no increase in statistical stability of received SNR. Fig. 8 shows the performance of the system when $L$ increases with $M$ and $L=M$. As evident, the outage-capacity curves become steeper as well. 


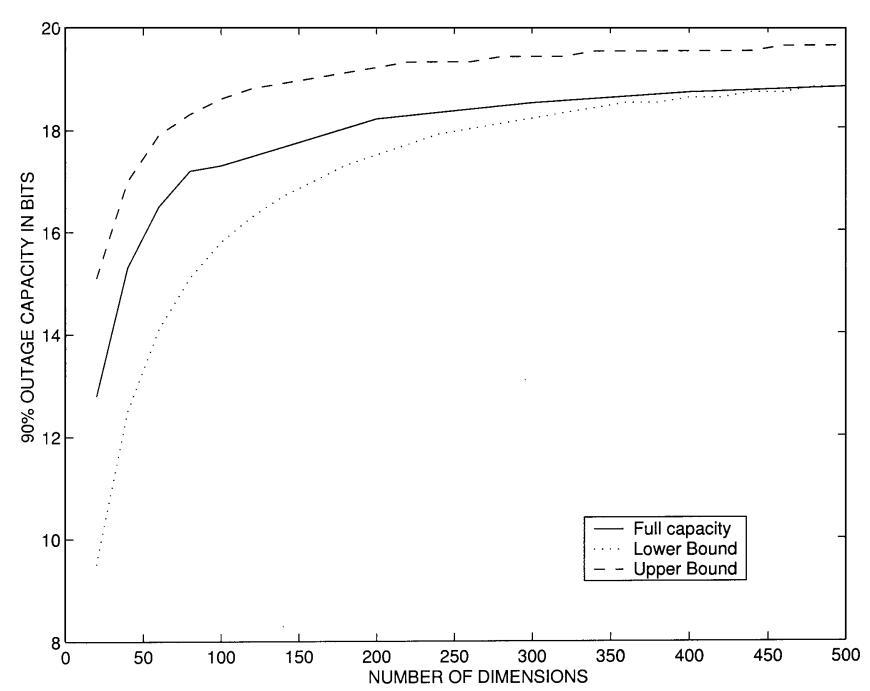

Fig. 9. Outage capacity for $(N, N)$ and $(M, N)$ systems.

The expression (43) provides a lower bound on the outage capacity of the full-dimensional multipath channel, and the bound becomes tighter as the dimensionality of the signaling space increases. An expression similar to (43) can be derived that is an upper bound on the outage capacity of the multipath channel. In the full-dimensional multipath channel, the signal is transmitted in all $N$ time samples. Each transmitted symbol undergoes dispersion across $L$ time samples. The dispersion destroys orthogonality between the transmitted signals and causes interference between them. A simple upper bound can be obtained by ignoring the effects of interference-all of the $N$ time samples encounter $L$ th-order diversity and do not interfere at the receiver. Such a system corresponds to $N$ parallel RAKE transceivers (and requires $N L$ signal-space dimensions at the receiver). A lower bound for the outage capacity expression for the system is exactly (43) with $N=L M$, and the upper bound is (43) with $N$ replaced by $N L$. Thus, for the $(N, N)$ multipath channel, we have that

$$
\begin{aligned}
\frac{\gamma\left(L, \frac{N}{P}\left(2^{R L / N}-1\right)\right)}{\Gamma(L)} & \leq \operatorname{Pr}(C<R) \\
& \leq \frac{\gamma\left(L, \frac{N L}{P}\left(2^{R / N}-1\right)\right)}{\Gamma(L)} .
\end{aligned}
$$

It is easy to show that the two bounds converge to the same value as $N \rightarrow \infty$. Fig. 9 shows that as $N$ increases, the outage capacity bounds in (44) closely approximate the capacity of the $(N, N)$ channel. The capacity curve for the channel was obtained through 10000 realizations. The reason the two bounds converge to the same value is that increasing the number of parallel channels in a system provides diminishing returns. Furthermore, for given $L$, the loss of dimensions due to separating transmitted signals by $L$ dimensions becomes negligible as $N$ increases. For example, in an AWGN channel, $90 \%$ of the capacity of the infinite bandwidth AWGN channel is achieved for $N \geq 4 P$ [14].

\section{Virtual Multiple-Antenna System}

This example illustrates using the virtual MIMO framework to facilitate application of space-time codes developed for mul- tiantenna systems to CDMA systems. We present a transceiver structure that transforms the multipath channel with $L$ degrees of freedom into a virtual antenna array system with $I$ transmitters, $J$ receivers, $I J=L$, and independent coupling between antenna pairs [15]. The transceiver is characterized in the time domain for simplicity. However, the transceiver can be applied to CDMA systems as well, since the transformations of Section IV can be used to transform a CDMA system into an equivalent time-domain system.

We refer to (14) again. The signal is transmitted in the first $I$ time samples, $\mathbf{x}^{t}=\left[x_{0}, x_{1}, \ldots, x_{I-1}, 0, \ldots\right]^{T}$. The received signal is in a space of $I+L-1$ dimensions, but the receiver looks at only the $J$ dimensions of $\mathbf{y}^{t}$ given by $\left[y_{I-1}, y_{2 I-1}, \ldots, y_{I J-1}\right]^{T}$. The resulting $I$-input $J$-output system is represented by

$\left[\begin{array}{c}\tilde{y}_{0} \\ \tilde{y}_{1} \\ \vdots \\ \tilde{y}_{J-1}\end{array}\right]=$
$\left[\begin{array}{cccc}h_{J-1} & h_{J-2} & \ldots & h_{0} \\ h_{2 J-1} & h_{2 J-2} & \ldots & h_{J} \\ \vdots & \vdots & \ldots & \vdots \\ h_{I J-1} & h_{I J-2} & \ldots & h_{(I-1) J}\end{array}\right]\left[\begin{array}{c}x_{0} \\ x_{1} \\ \vdots \\ x_{I-1}\end{array}\right]+\left[\begin{array}{c}\tilde{w}_{0} \\ \tilde{w}_{1} \\ \vdots \\ \tilde{w}_{J-1}\end{array}\right]$

where $\tilde{y}_{j}=y_{I(j+1)-1}, \tilde{w}_{j}=w_{I(j+1)-1}$ for $j=0,1, \ldots, J-1$. Note that the $J \times I$ channel matrix $\mathbf{H}$ contains uncorrelated, complex Gaussian entries. The system can be viewed as an $I$-transmit $J$-receive multiple-antenna system with i.i.d. Rayleigh fading matrix channel. Existing space-time codes (e.g., see [4]) can be directly applied to this transceiver and, after suitable transformations (Fig. 3), to CDMA transceivers. An expression for the ergodic capacity of multiple-antenna systems is given in [3]. The ergodic capacity of this channel is given by [3]

$$
\begin{aligned}
\int_{0}^{\infty} \log _{2}\left(1+\frac{P \lambda}{I}\right) \sum_{k=0}^{T_{\min }-1} \frac{k !}{\left(k+T_{\max }-T_{\min }\right) !} \\
\\
{\left[L_{k}^{T \max -T_{\min }}(\lambda)\right]^{2} \lambda^{T_{\max }-T_{\min }} e^{-\lambda} d \lambda }
\end{aligned}
$$

where $T_{\min }=\min (I, J), T_{\max }=\max (I, J)$, and $L_{m}^{n}(\cdot)$ is the generalized Laguerre polynomial of order $m$ with parameter $n$.

We now compare this virtual MIMO transceiver with the RAKE transceiver of Section VI-A. The virtual MIMO transceiver creates an $(I, J)$ system that has $\min (I, J)$ parallel channels. It provides clear capacity gains over the RAKE transceiver due to an increase in the number of parallel channels. Fig. 10 illustrates the performance of both transceivers. At the $1 \%, 5 \%$, and $10 \%$ outage probability levels and for high SNR (larger than $20 \mathrm{~dB}$ ), the improvement in performance of the virtual MIMO transceiver over the RAKE transceiver for $I=2, J=2, L=4$ is almost $5 \mathrm{~dB}$. The improvement in performance for $I=2, J=3, L=6$ is more than $7 \mathrm{~dB}$ (not shown in Fig. 10).

We note in passing that using circularly shifted versions of a spreading waveform as a basis for the signal space is key to the application of existing space-time codes to CDMA systems. 


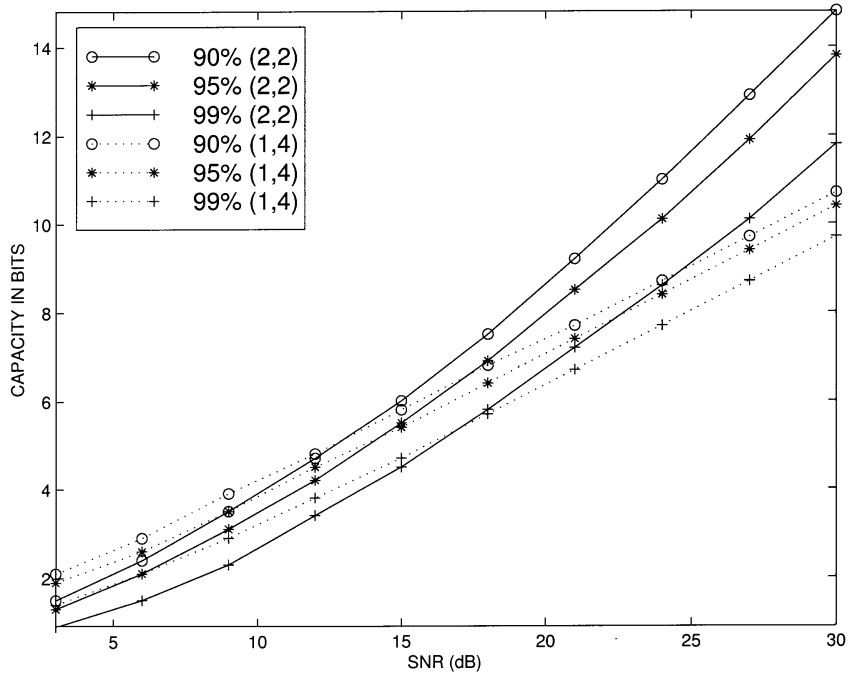

Fig. 10. Outage-capacity curves for $(1,4)$ and $(2,2)$ virtual MIMO systems.

The reason is that space-time codes, originally designed for multiple-antenna systems, exploit the structure of the channel statistics at the transmitter without requiring knowledge of the channel realizations. Our example shows that the same channel matrix structure can be obtained over single-transmitter singlereceiver multipath channels using particular temporal signaling schemes. By appropriate transformations, these transceivers can also be applied to CDMA systems. In general, to do these transformations at the transmitter, i.e., to do the preprocessing at the transmitter to obtain a certain structure in the effective channel matrix, the transmitter needs to be aware of the eigenvectors of the channel matrix. Transmitting on circularly shifted versions of a spreading waveform preserves the structure and eigenfunctions of the channel, so that this preprocessing depends only on the choice of spreading code $(\mathbf{C})$ and not on the actual channel realizations.

\section{E. Application of OFDM Codes to CDMA Systems}

This example briefly illustrates how OFDM codes can be applied to CDMA systems. Starting with the wideband CDMA model, the transformations detailed in Fig. 4 can be used to effectively produce a frequency-domain system. Denote the input vector to this new $(N, N)$ system by $\mathbf{x}^{f}=\left[x_{0}^{f}, x_{1}^{f}, \ldots, x_{N-1}^{f}\right]$. When the channel offers $L$ th-level diversity, $(N / L)$ different information symbols can be transmitted, with each information symbol achieving $L$ th-order diversity. This can be done in a simple way as follows. The first symbol is transmitted in the following coordinates of $\mathbf{x}^{f}: x_{0}^{f}, x_{N / L}^{f}, \ldots, x_{N(L-1) / L}^{f}$. Simiarly, each of the other symbols are transmitted in exactly $L$ coordinates, the different coordinates separated by $(N / L)$ samples, and the different symbols are offset by one coordinate. This is a well known and simple OFDM coding scheme that achieves the full diversity afforded by the channel. The novelty of this transceiver lies in the fact that OFDM codes can be applied to CDMA transceivers that are already coupled to the channel.

\section{CONCLUDING REMARKS}

In this paper, we develop a virtual MIMO framework for characterizing single-transmitter single-receiver multipath fading channels, where the virtual multiple inputs and outputs are created by dimensions of the signaling scheme. The essence of the framework is a representation of the system with respect to appropriately chosen basis functions for the signal space. The proposed framework has many advantages. First, it provides a general approach for studying the interaction between the signal space and the channel and unifies existing systems. Second, it suggests a method for design of novel transceivers by modifying existing ones. For example, it suggests a method for application of codes developed for OFDM systems and space-time codes developed for multiple-antenna systems to existing CDMA systems. Finally, it greatly facilitates ergodic and outage-capacity analysis. In particular, by analyzing particular transceivers for the multipath channel, we are able to provide new expressions that bound the outage-capacity performance of the channel. Work in progress extends these results to the case of time-varying channels [16], [17]. Another line of research worth pursuing and a useful extension to these results is a framework that facilitates transceiver analysis and design in the multiuser case.

\section{REFERENCES}

[1] J. G. Proakis, Digitial Communications, 3rd ed. New York: McGrawHill, 1995.

[2] G. J. Foschini, "Layered space-time architecture for wireless communication in a fading environment when using multi-element antennas," Bell Labs Tech. J., vol. 1, no. 2, pp. 41-59, 1996.

[3] E. Telatar, "Capacity of Multi-Antenna Gaussian Channels," AT\&T-Bell Labs Internal Tech. Memo., 1995.

[4] V. Tarokh, N. Seshadri, and A. R. Calderbank, "Space-time codes for high data rate wireless communication: Performance criteria and code construction," IEEE Trans. Inform. Theory, pp. 744-765, Mar. 1998.

[5] L. H. Ozarow, S. Shamai, and A. D. Wyner, "Information theoretic considerations for cellular mobile radio," IEEE Trans. Veh. Technol., vol. 43, pp. 359-378, May 1994.

[6] E. Biglieri, J. Proakis, and S. Shamai (Shitz), "Fading channels: Information-theoretic and communications aspects," IEEE Trans. Inform. Theory, pp. 2619-2692, Oct. 1998

[7] P. A. Bello, "Characterization of randomly time-variant linear channels," IEEE Trans. Commun. Syst., vol. CS-11, pp. 360-393, Nov. 1963.

[8] D. Slepian, "On bandwidth," Proc. IEEE, vol. 64, pp. 292-300, Mar. 1976.

[9] R. Gallager, Information Theory and Reliable Communication. New York: Wiley, 1968, ch. 8: Waveform Channels.

[10] U. Grenander and G. Szego, Toeplitz Forms and Their Applications. New York: Chelsea, 1983.

[11] R. M. Gray, "On the asymptotic eigenvalue distributions of Toeplitz matrices," IEEE Trans. Inform. Theory, vol. IT-18, pp. 725-729, Nov. 1972.

[12] D. Rajan, E. Erkip, and B. Aazhang, "New spread spectrum techniques for multiple antenna transmit diversity," in Proc. Global Telecommunications Conf., 1999, pp. 2321-2325.

[13] R. A. Horn and C. R. Johnson, Topics in Matrix Analysis. Cambridge, U. K.: Cambridge Univ. Press, 1991

[14] J. L. Massey, "Toward an information theory of spread-spectrum systems," in Code Division Multiple Access Communications, S. Glisic, Ed. Boston, MA: Kluwer, 1995, pp. 29-46.

[15] A. Ganesan and A. M. Sayeed, "Bandwidth-efficient exploitation of the degrees of freedom in a multipath fading channel," in Proc. IEEE Int. Symp. Information Theory, June 2000, p. 161.

[16] T. A. Kadous, K. Liu, and A. M. Sayeed, "Optimal time-frequency signaling for rapidly time-varying channels," in Proc. Int. Conf. Acoustics, Speech and Signal Processing, May 2001, pp. 2405-2408.

[17] K. Liu, T. A. Kadous, and A. M. Sayeed, "Orthogonal time-frequency signaling for doubly dispersive channels," IEEE Trans. Inform. Theory, submitted for publication. 